\title{
Dense Plasmas Research in the Chilean Nuclear Energy Commission: Past, Present and Future
}

\author{
Leopoldo Soto, Patricio Silva, José Moreno, Marcelo Zambra, \\ Gustavo Sylvester, Andrey Esaulov† and Luis Altamirano ${ }^{\ddagger}$ \\ Comisión Chilena de Energía Nuclear \\ Casilla 188-D, Santiago, Chile
}

Received on 26 June, 2001

\begin{abstract}
A review of the dense transient plasmas researches, developed in the Chilean Nuclear Energy Commission, is presented. A brief summary of the researches done in collaboration with the Pontificia Universidad Católica de Chile, between 1993 to 1997, is shown. In addition, the program "Plasma Physics in Small Devices", developed at the Comisión Chilena de Energía Nuclear since 1999 is delineated. The diagnostics development and results obtained during three experiments using small pinch devices are shown: a capillary discharge; a $\mathrm{Z}$ pinch driven by a small generator; and a low energy plasma focus. The experiments were complemented by magnetohydrodynamics numerical calculations, in order to assist the design and physical interpretation of the experimental data. The diagnostics techniques used in these experiments include current and voltage monitors, multipinhole camera, plasma image using a ICCD camera gated from 3 to $20 \mathrm{~ns}$, holographic interferometry, and vacuum ultraviolet spectroscopy. Recently, the pulse power generator SPEED 2, a medium energy and large current device $\left(187 \mathrm{~kJ}, 4 \mathrm{MA}, 300 \mathrm{kV}, 400 \mathrm{~ns}, \mathrm{dI} / \mathrm{dt} \sim 10^{13} \mathrm{~A} / \mathrm{s}\right)$, has been transferred from the Düsseldorf University to the Comisión Chilena de Energía Nuclear. Future experiments, and the perspectives of using this device, are also discussed.
\end{abstract}

\section{Introduction}

The dense z-pinch offers a compact and direct method of achieving very high energy density plasmas of relevance for controlled fusion and for very intense X-ray sources $[1,2]$. It is important to note the renewed interest in z-pinches research as soft X-ray sources for thermonuclear inertial confinement devices [3]. Under this approach, the z-pinch is not a dense plasma thermonuclear reactor, as expected during decades. The pinch provides a very intense soft X-ray source, which irradiates a deuterium-tritium target. Active research on z-pinches is currently ongoing at several laboratories in the USA, England, Germany, Russia and elsewhere [4-9]. These experiments are carried out in large devices, like the one at Sandia National Laboratory in the US, in a huge generator capable of delivering 20MA in $100 \mathrm{~ns}\left(d I / d t>10^{14} \mathrm{~A} / \mathrm{s}\right)$ into the plasma. Similar large facilities are available at TRINITI in Russia.

The z-pinch is a fascinating object, yet an open field, whose behaviors are determined by a variety of magnetohydrodynamics processes, radiative transport, atomic physics, plasma microinstabilities, and beam physics [1, 2]. Plasma Physics research at the Chilean Nuclear Energy Commission (CCHEN) started towards the end of 1993. During the period considered between the end of 1993 to 1997, the work was done in collaboration with the Plasma Physics Group of the Pontificia Universidad Católica de Chile (PUC). While initiating the development of plasma diagnostics, and the construction of experiments in small devices at CCHEN, the experimental facilities at PUC were used. Thus, in few years, an active research group was born at CCHEN. Transient dense plasmas like z-pinches have been studied, both experimentally and theoretically, at CCHEN in the frame of the research program "Plasma Physics in Small Devices" [10].

Small plasma pinches can reproduce scenarios of high energy densities, radiation emission, and instability phenomena, which trigger fundamental and applied research with relatively low costs. A dynamic pinch is a transient plasma column conducting electrical current, which is confined by the associated magnetic field. Plasma pinches usually require the use of high

\footnotetext{
*E-mail: lsoto@cchen.cl

†Present address: Institute of Theoretical and Experimental Physics, Moscow 117259, Russia, and Laboratory for Plasma Astrophysics, Toyama University, 3190 Gofuku, Toyama 930-8555, Japan.

$\ddagger$ Permanent address: Dicontek, Pasaje Galicia 1365, La Florida, Santiago, Chile
} 
pulsed voltages ( $\mathrm{kV}$ to $\mathrm{MV}$ ) and high currents ( $\mathrm{kA}$ to MA). Generally, these devices operate by connecting a charged capacitor array to a pair of electrodes. Different electrode configurations have been proposed to create plasma pinches during pulsed discharges, being z-pinch, $\theta$ pinch and plasma focus the best-known.

The applications of pinch discharges are mainly related to the radiation emitted by the plasma, namely electrons and ion beams, $\mathrm{X}$ rays, and neutrons (using deuterium). Fast electrical discharges are also used to study plasma dynamics and stability. The development and application of diagnostics, with enough temporal and spatial resolution, are required to study dense transient plasmas. In this article, diagnostics techniques, instruments and experiments in the frame of the research program "Plasma Physics in Small Devices" of the CCHEN are shown. Three experiments are discussed, which use small devices to study the fundamentals and applications of transient pinch electrical discharges: a) vacuum ultraviolet (VUV) to soft $\mathrm{x}$-ray radiation from a fast capillary discharge, b) the early stages, dynamics and stability of a gas embedded $\mathrm{Z}$ pinch, and c) the development of a neutron pulsed source based on a small plasma focus. Recently, the pulse power generator SPEED 2, a medium energy and large current device (187kJ, 4MA, 300kV, 400ns, dI/dt $\sim 10^{13} \mathrm{~A} / \mathrm{s}$ ) [11], has been transferred from the Düsseldorf University to the Comisión Chilena de Energía Nuclear. Future experiments, and the perspectives of using this device, are also discussed.

\section{Plasma diagnostics and in- strumentation}

Transient electrical discharges like z-pinches can produce plasmas with sizes varying from centimeters to hundred of microns, with inhomogeneities of tens of microns or less. Particle density from $10^{23}$ to $10^{26} \mathrm{~m}^{-3}$. Temperatures from tens of $\mathrm{eV}$ to several $\mathrm{keV}$. The life time of this plasmas are in the order of some nanoseconds to hundred of nanoseconds with fast processes that can occur in nanoseconds or sub nanoseconds scales. Soft and hard x-ray are emitted. Particles as electrons, ions and neutrons, can be emitted. High spatial and temporal resolution diagnostics are required. In order to obtain the maximum benefit from the experimental devices, proper instruments and diagnostics must be developed. An important goal of the plasma physics group of the CCHEN has been to develop plasma diagnostics and instrumentation.

Electrical diagnostics. Voltage, total current and current derivative are measured with usual monitors, resistive and capacitive divider, and Rogowskii coil located close to the plasma load.

Images from the visible plasma ligth. An intensified CCD camera (ICCD) gated from 3 to 20 ns and syn- chronized with the discharge has been implemented in order to obtain plasma images from the visible light emitted. Combining this diagnostics with filters, broad band spectroscopy can be done. Fig. 1 shows an image of a very small plasma focus experiment at CCHEN. The radial compression of the plasma occurring after the current sheaths reaches the axial end of the electrodes can be seen. In a next section, details of the plasma focus experiment will be described.

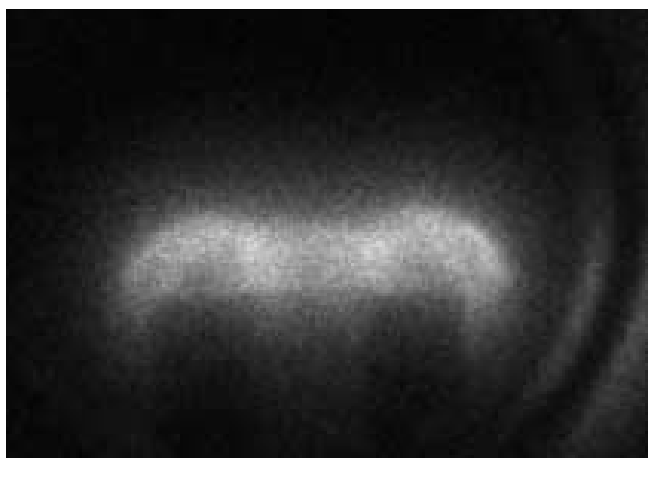

\section{$20 \mathrm{~mm}$}

Figure 1. A plasma image obtained with a ICCD camera gated at $10 \mathrm{~ns}$ is shown. The plasma was obtained from a very small plasma focus experiment at CCHEN operating in $\mathrm{H}_{2}$ at $1 \mathrm{mb}, 25 \mathrm{kV}$ charging voltage and $50 \mathrm{~J}$ storage energy in the capacitor bank. The radial compression of the plasma occurring after the current sheths reaches the axial end of the electrodes can be seen.
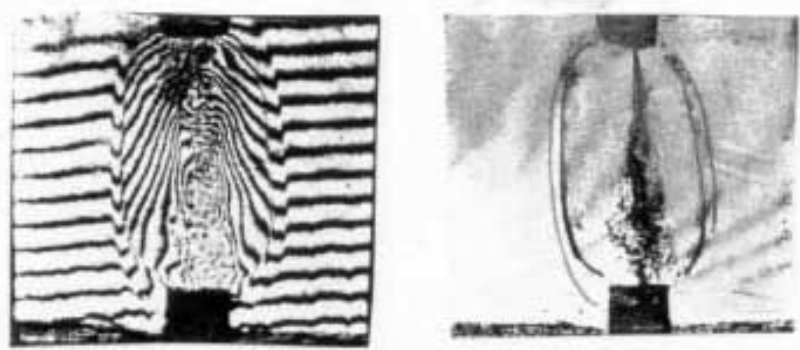

Figure 2. Simultaneous single shot image-plane holographic interferometry and shadowgraphy. The images corresponds to a gas embedded compressional z-pinch [refs. 14 and 15]. The distance between electrodes is $10 \mathrm{~mm}$. The high shift deviation of the fringes in the interferogram is according with the dark central core in the image-plane shadowgram.

Optical refractive diagnostics. It is well known that the density of the plasma components is related with the refraction index of the plasmas. Therefore, optical refractive diagnostics as shadowgraphy, schlieren, and inteferometry are very useful to obtain the size, electron density, dynamics and stability behavior of the plasma. Pulsed diagnostics has been developed at the PUC plasma laboratory. A frequency doubled NdYAG laser pulse (6ns) was used for optical diagnostics. 
Simultaneous single shot image-plane holographic interferometry and shadowgraphy [12], and multiframe holographic interferometry ( 8 frames separated by 10 ns between them) [13] was implemented and used to study different kinds of gas embedded z-pinches and vacuum spark discharges [14-18]. Simultaneous single shot image-plane holographic interferometry and shadowgraphy provide good spatial resolution (Fig. 2). On the other hand, the multiframe holographic interferometry system use Fresnel holography, thus providing a good temporal resolution in spite of spatial resolution (Fig. 3). Both techniques are complementary. Fig. 2 shows a shadowgram and an image plane interferogram obtained simultaneously in a needle initiated gas embedded z-pinch discharge. Fig. 3 shows an eight frames sequence of a laser initiated gas embedded z-pinch, note that the $m=1$ instability transforms the plasma column in a helix in less than 10ns. The multiframe holographic system requires a lot of opto-mechanical components; original and low cost mechanical mounts for mirrors and beam-splitter were designed and constructed for this and other similar purposes [19].

In the particular case of vacuum spark discharges small-scale inhomogeneities develop [16-18]. The size of these high-density regions is of the order of $\sim 10-100 \mu \mathrm{m}$, embedded in a plasma column of $\sim 500 \mu$ m diameter of lower density. Several holographic schemes have been suggested to study microscopic objects. However, in all of these procedures the observation of small-scale structures precludes the simultaneous observation of larger scale inhomogeneities. A procedure by which simultaneous large and small scale inhomogeneities of the refractive index of an object has been developed at CCHEN [20]. The information is obtained from a hologram and a simultaneous double exposure holographic interferogram. Small scale information is obtained by observing under magnification the interference patterns which form the actual hologram. This is completed by observing the reconstruction of the same hologram. The large scale information is obtained by the reconstruction of the double exposure holographic interferogram. Fig. 4 shows the result when the technique was applied to a laser produced spark in air. At early times small scale structure is present in this type of microplasma. This application was done using a frequency doubled Nd-YAG laser pulse. The method can be directly applied to obtain the density profiles of hot-spots developed in a transient plasma discharge [21].

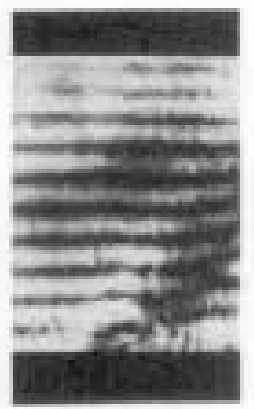

Sns

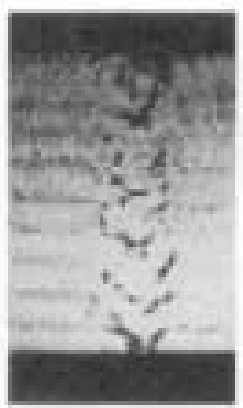

$45 n s$
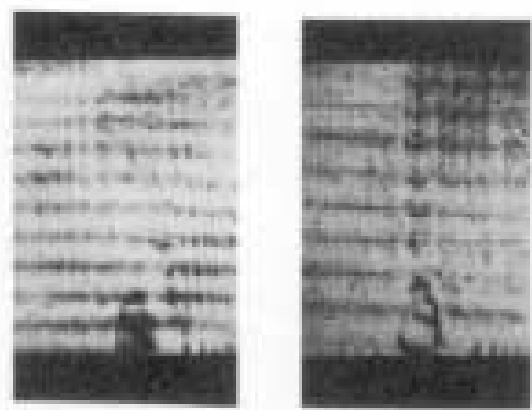

$15 n s$

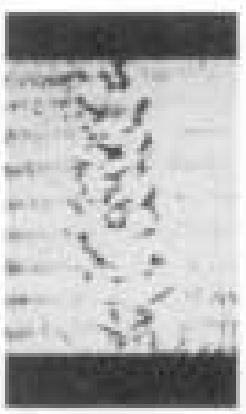

$55 n s$

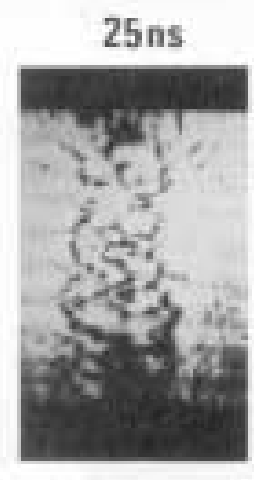

$65 n s$

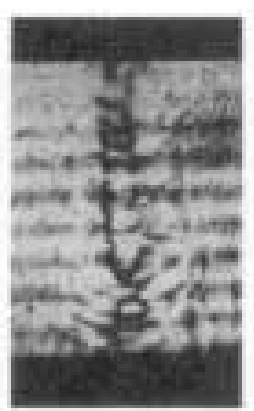

$35 \mathrm{~ns}$

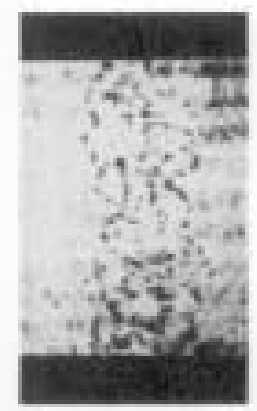

$75 n s$

Figure 3. Eight frames sequence of interferograms of a laser initiated gas embedded z-pinch, note that the $m=1$ instability (at $35 \mathrm{~ns}$ ) transforms the plasma column in a helix in less than 10ns [ref. 13]. 


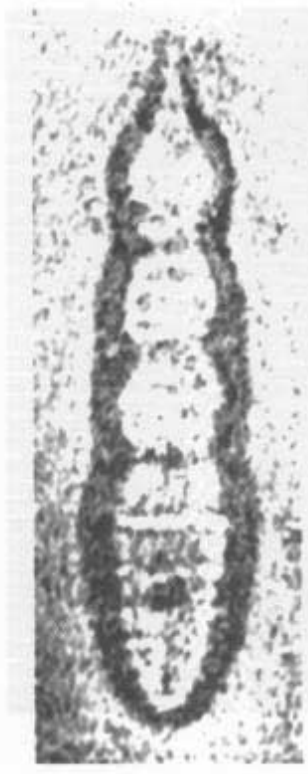

(a)

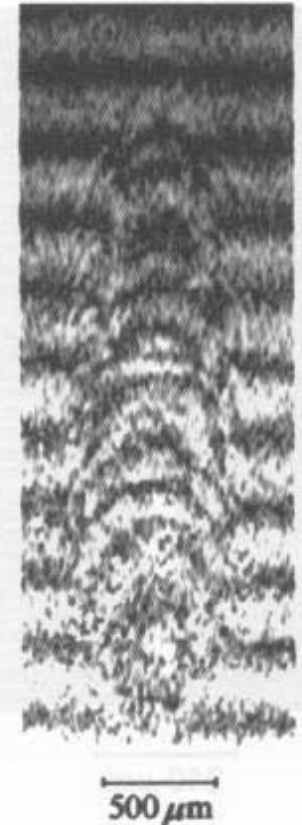

(b)

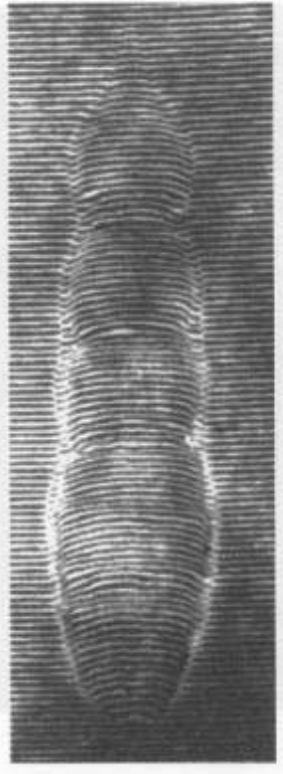

(c)

Figure 4. Images obtained with a micro-interferometry technique [ref. 20]. The three images show (a) image of the first order from the transmission hologram; (b) the double-exposure holographic interferogram; and c) magnified fringe pattern of the transmission hologram, the interferogram of the object contained in the hologram (micro-interferogram). It is clearly evident that the fine-scale features observed in the micro-interferogram are lost in the double-exposure interferogram.

At present, CCHEN does not count with a pulsed laser, thus it is not possible to apply in this installation the developed optical refractive diagnostics for the present experiments (capillary discharge and plasma focus). However, an alternative scheme has been implemented and it is ready to be applied. Using an ICCD camera gated from 3 to $20 \mathrm{~ns}$ in combination with a continuos He-Ne laser, optical refractive diagnostics as shadowgraphy, schlieren and interferometry are obtained.

VUV to soft X-ray diagnostics. VUV to soft X-ray radiation from dense plasmas are nowadays interesting for studies and applications related to: a) inertial confinement for thermonuclear fusion studies and b) physical mechanisms related to laser emission in the VUV to soft X-ray region in a table-top device. A series of diagnostics in order to study the VUV radiation from a fast and small hollow cathode capillary discharge (some centimeter length, $\sim 1 \mathrm{~mm}$ diameter, $\sim 5 \mathrm{kA}$ achieved in $\sim 5 \mathrm{~ns})$ has been developed and applied at CCHEN [22, 23].

A coaxial cable was biased at $-200 \mathrm{~V}$ and located at the discharge axis, behind the anode, in order to detect electrons (negative signal) or photons with energy above $4.3 \mathrm{eV}$ (positive signal, like a $\mathrm{X}$ ray diode). A multipinhole camera with a magnification of $m=3$, attached to a microchannel plate (MCP) sensitive to $\mathrm{X}$ rays and VUV radiation is used to register 1 frame every 4 ns (Fig. 5). VUV spectra of the capillary discharge have been obtained by means of a grazing incidence spectrograph GISVUV1-3S (Fig. 6). A gold-coated replica grating of 300 lines $/ \mathrm{mm}$, with 1 -m curvature radius, was used. The grazing angle was $4^{\circ}[22,23]$. Results obtained in a fast and small hollow cathode capillary discharge are shown in a next section.

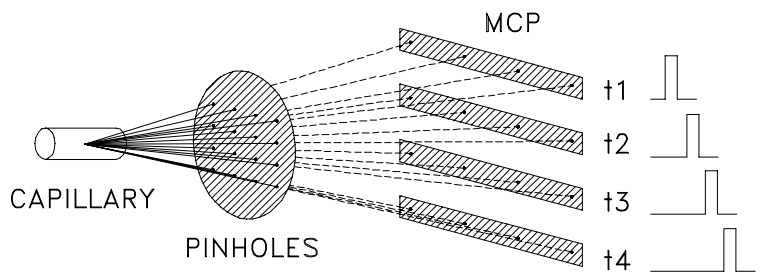

Figure 5. Multipinhole camera. A four frame microchannel plate (MCP) was used to register images: one frame every $4 \mathrm{~ns}$. The MCP is sensitive only to X-ray and VUV radiation. The gating pulse is $5 \mathrm{~ns}$. A square array of 16 pinholes was used; 4 columns with equal size pinholes per column: $50,100,200$ and $300 \mu \mathrm{m}$, respectively. The four rows or the pinhole array correspond to different frames separated by $4 \mathrm{~ns}$. So, time-space resolution plasma images can be obtained. 


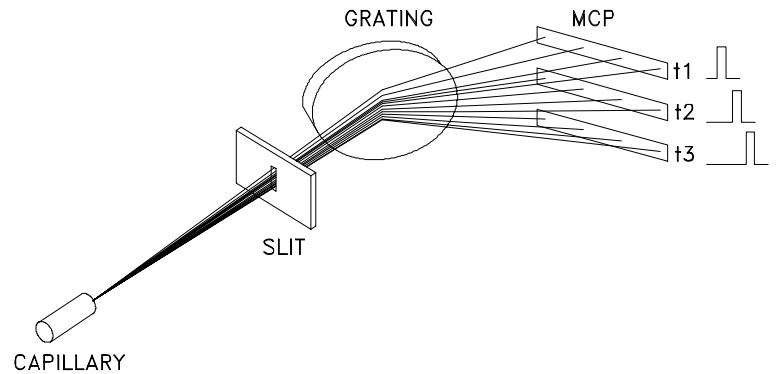

Figure 6. VUV spectrograph. VUV spectra of the capillary discharge has been obtained by means of a grazing incidence spectrograph GISVUV1-3S. Gold-coated replica grating of 300 lines $/ \mathrm{mm}$, with a curvature radius of $1 \mathrm{~m}$ was used. The grazing angle was $4^{o}$. The spectra was registered in the same four MCP frames used with the pinhole camera (4ns between frames and $5 \mathrm{~ns}$ gating pulse). Due to the geometry defined by the spectrograph, only three frames are used.

Pulsed Power Technology. Voltages of the order of kilovolts to megavolts with rise time of the order of nanoseconds to microseconds are used to drive experiments in dense plasmas as z-pinches, plasma focus, capillary discharges, etc. Pulsed power technology relies on power multiplication through time compression. The first stage consists on a capacitor bank, which can be charged up slowly. In several devices the capacitor bank is a Marx generator in order to allow voltage multiplication. The second stage is a pulse forming circuit, typically a water transmission line, which charges up very rapidly and it is then switched to deliver a fast pulse of high power to load. This kind of generator is characterized by a fast rise time in the current at the load, typically tens of nanosecons and maximum currents of hundreds of kiloamperes or megamperes. Marx generators used for voltage multiplication consist of a set of capacitors which are charged up in parallel and then stacked up in series by a number of switches to achieve voltage multiplication. A pulse voltage multiplier without switches was developed at CCHEN [24]. The pulse voltage multiplier device consists in a set on " $n$ " transmission lines connected in parallel at the entry and connected in series at the end. A scheme of the device is shown in Fig. 7. Fig. 8 shows the results in a practical demonstration of the device in which coaxial cables RG231 were used. For the authors it is important to note that there is a practically identical device that was developed previously [25] and independently of the reference [24].

To the diagnosis capability achieved at the CCHEN, we will add in the near future: 1 ns time resolution holographic interferometry with a $\mathrm{N}_{2}$ laser; 2 dimensional optical multichannel analyzer; time integrated and temporal resolution neutrons detection; time resolved broad band emission using filtered PIN diode arrays; and time resolved X-ray spectroscopy.

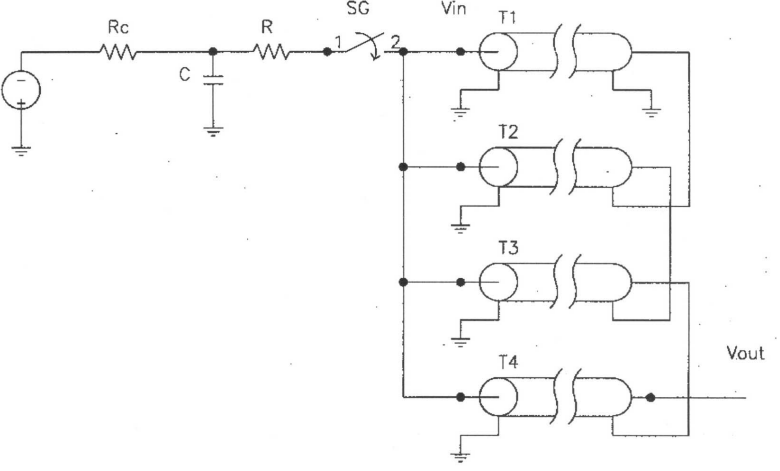

Figure 7. Schematic circuit of a pulse voltage multiplier [ref. 24]. In a practical realization $\mathrm{Rc}=100 \mathrm{M} \Omega$, SG: spark gap, $\mathrm{R}=3.3 \Omega, \mathrm{C}=7 \mathrm{nF}$. The impedance of each line is $\mathrm{Z}=50 \Omega$ (RG231 coaxial cables), and the length is $2 \mathrm{~m}$, i.e., a transient time of the transmission line equal to $10 \mathrm{~ns}$.

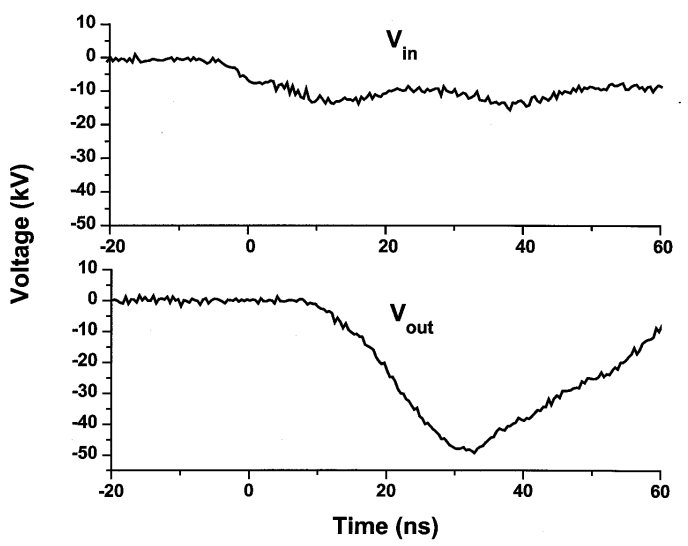

Figure 8. An output voltage with a peak of $50 \mathrm{kV}$, with an input voltage of $13 \mathrm{kV}$ is obtained with the pulse voltage multiplier shown in Fig. 7. The rise time at the output is less than $20 \mathrm{~ns}$.

\section{VUV radiation from capil- lary discharges}

In order to produce high brightness radiation with an electrical discharge, high energy densities should be provided to the plasma, $\sim 10^{12} \mathrm{~J} / \mathrm{m}^{3}$ (i.e. some MJ into a plasma column of a centimeter in length and radius). This is usually done by means of huge facilities such as the Sandia National Laboratory in the USA [1-6]. Intermediate devices have been used to produce vacuum ultraviolet (VUV) to soft $\mathrm{X}$ ray radiation [26] the most well-known being soft X-ray lasing [27, 29]. Interestingly, $10^{12} \mathrm{~J} / \mathrm{m}^{3}$ can also be provided using small devices. In effect, by confining the plasma to submilimetric volumes, less than $1 \mathrm{~J}$ is required to reach high energy densities (as an extreme example $0.1 \mathrm{~J}$ is enough for a $60 \mu \mathrm{m}$ diameter sphere). The key point to produce those conditions in small devices is the current 
rate, which should reach about $10^{13} \mathrm{~A} / \mathrm{s}$ [30]. Consequently, the inductance should be very low.

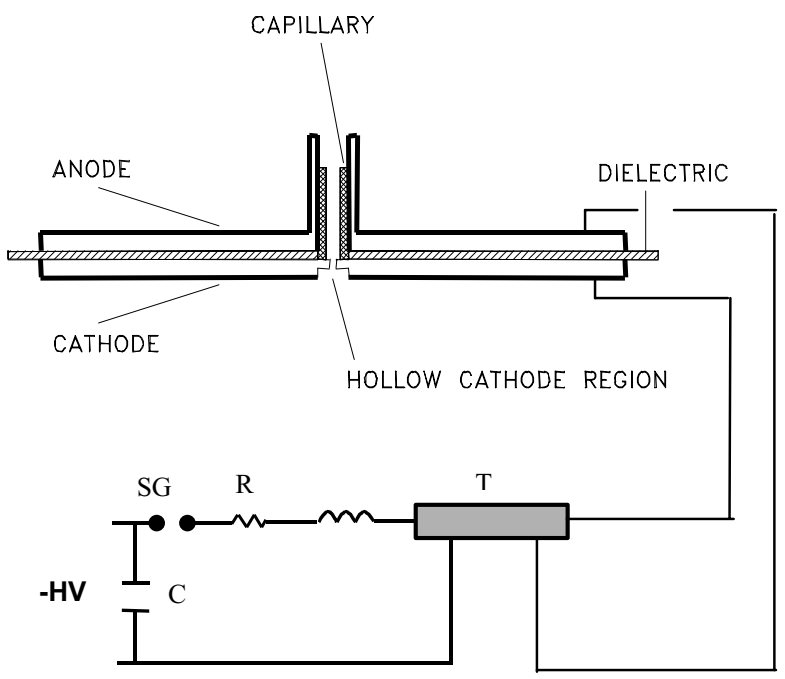

Figure 9. Capillary discharge device. A pair of 90mm diameter brass electrodes form the anode and the cathode of the discharge, as well as a parallel plate capacitor. A capillary of sub-millimeter radius and with a length of some centimeters, is placed at the axis. The discharge is produced inside the capillary between the capacitor plates. To operate the discharge, a direct current charged primary capacitor of $\mathrm{C}=7 \mathrm{nF}$ is used to charge the storage capacitor with a pulse. From the spark gap SG, the capacitor of the main discharge is connected to three coaxial cables in parallel $(\mathrm{T})$.

We are studying the physical mechanisms related to laser emission in the VUV to soft $\mathrm{X}$-ray region in a table-top device. A fast capillary discharge was constructed at the Comisión Chilena de Energía Nuclear, CCHEN, following the design reported by Choi and Favre $\left(0.1-1 \mathrm{~J}, d I / d t \geq 10^{12} \mathrm{~A} / \mathrm{s}\right)$ [31]. The apparatus is shown in Fig. 9. A pair of $90 \mathrm{~mm}$ diameter brass electrodes form the anode and the cathode of the discharge, as well as a parallel plate capacitor. The insulator (Mylar and polyvinylidenefluoride, PVDF) between the electrodes is also the capacitor dielectric. A 1 - $1.5 \mathrm{~mm}$ inner diameter capillary tube, $0.8,2.5,5.0 \mathrm{~cm}$ long, is placed at the axis. The discharge is produced inside the capillary. A primary $7 \mathrm{nF}$ capacitor charges the storage capacitor by means of a pulse. The length of the cable connections between the spark gap and the capacitor of the main discharge is calibrated to obtain the required timing between the discharge and the diagnostics. The ionization in the interelectrode space is assisted by a high-energy electron beam, which is originated in plasma inside the hollow cathode region [3133]. The discharge operates in Argon with a cathode pressure of 0.1-1.0 mbar. The anode pressure is around five times less than the cathode pressure. Current rates about $10^{12} \mathrm{~A} / \mathrm{s}$ were obtained applying voltages of 10 $\mathrm{kV}$.

The voltage in the capillary capacitor, the current and the current derivative were measured in our experiments. The VUV diagnostics described in the previous section II were applied to the discharge.

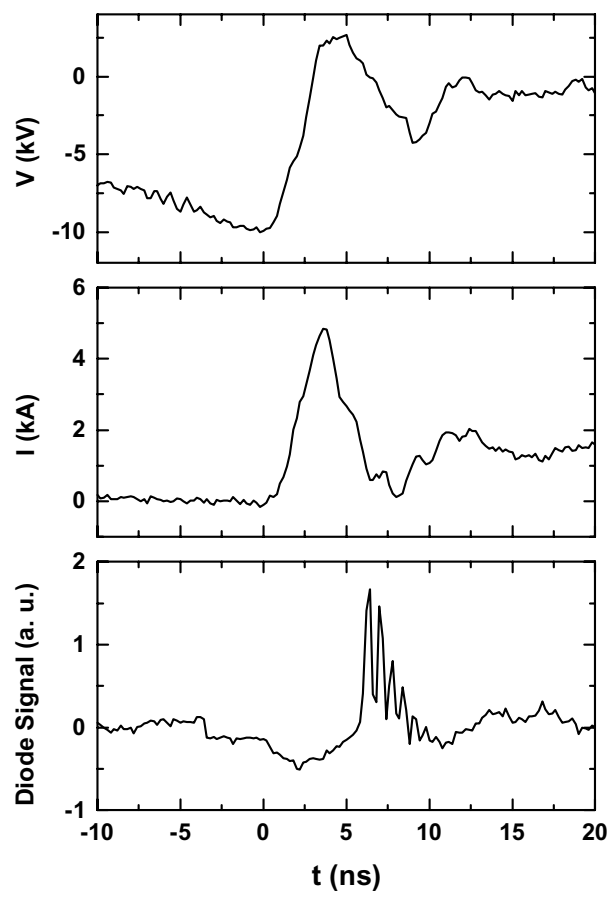

Figure 10. Signals of voltage, V, current, I, and from a $-200 \mathrm{~V}$ biased diode detector corresponding to a capillary discharge in argon at 500 mtorr cathode pressure, $8 \mathrm{~mm}$ capillary length and $1 \mathrm{~mm}$ diameter. Time $\mathrm{t}=0$ correspond to the breakdown that was at $-10 \mathrm{kV}$. The maximum current achieved was $5 \mathrm{kA}$ with a rise time $10-90 \%$ less than $3 n s$. From the diode detector a negative signal appears before the breakdown, corresponding to the electron beam. Later, a positive signal appears corresponding to the photonic radiation impacting on the diode, $3 \mathrm{~ns}$ after the peak current.

Fig. 10 shows typical signals of voltage, current and diode detector of a discharge along $8 \mathrm{~mm}$ length capillary and 0.650 mbar Argon cathode pressure, at approximately $-10 \mathrm{kV}$ charging voltage. Time $\mathrm{t}=0$ indicates the breakdown that occurs at $-10 \mathrm{kV}$. The peak current achieved was $5 \mathrm{kA}$ in 3.5 ns, i.e. a half period of $7 \mathrm{~ns}$ in agreement with the approximately $2 \mathrm{nH}$ inductance of the $8 \mathrm{~mm}$ long capillary. With a capillary three times longer $(25 \mathrm{~mm})$ the half period of the discharge increases by $70 \%$, to $6 \mathrm{~ns}$, in accordance to an inductance three times larger, demonstrating that the pulsed power has an appropriately low inductance on the order of $\mathrm{nH}$. Negative signals were observed in the diode detector before breakdown (Fig. 10), indicating electron beams. About 3 ns after the peak current there were indications of photon radiation impacting on the diode, which produced positive signals. Pinhole images taken during a discharge along an $8 \mathrm{~mm}$ length capillary and 0.650 mbar are shown in Fig. 11. It is important to note that the pinholes are not in the capillary axis, so 
some considerations must be taken into account. From simple geometry it is clear that images created from opposite ends of the capillary have different magnifications, and are centered in different position. Thus, for a qualitative analysis three possibilities were considered. For a radial homogeneous plasma inside the capillary, the image corresponds to non concentric circles (Fig. 12a). In a hollow plasma column, the image has a more complex structure (Fig. 12b). In a compressed plasma, the image becomes a line (Fig. 12c) [34]. According to that interpretation, in Figure 11, the images for $5 \mathrm{~ns}$ (2ns after peak current) suggest the existence of a homogeneous plasma column. For the $\Phi=50 \mu \mathrm{m}$ pinhole diameter, a central brightness core of $\sim 300 \mu$ m can be observed, which is not necessarily a compressed plasma.

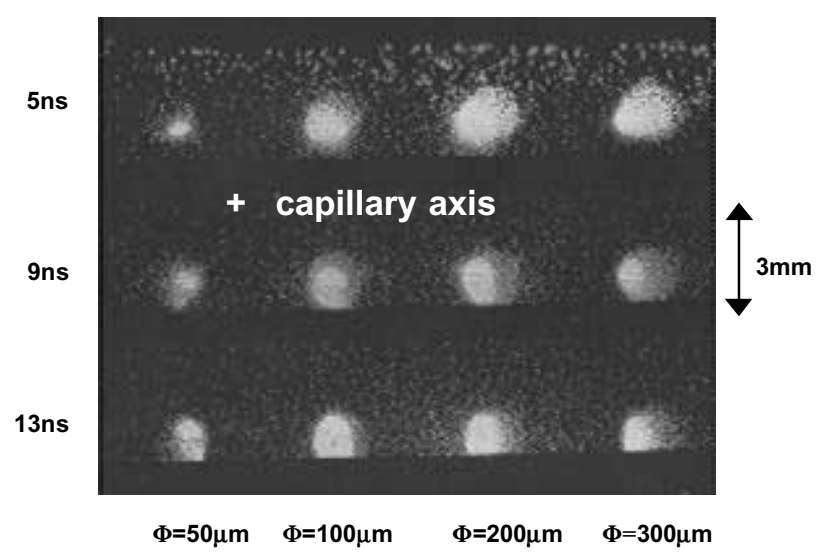

Figure 11. Pinhole images corresponding to a capillary discharge at 500 mtorr cathode pressure, a capillary of $8 \mathrm{~mm}$ in length and $1 \mathrm{~mm}$ in diameter. The cross indicates the position of the capillary axis. Unfortunately, only 3 of the 4 frames of MCP were working at that time.
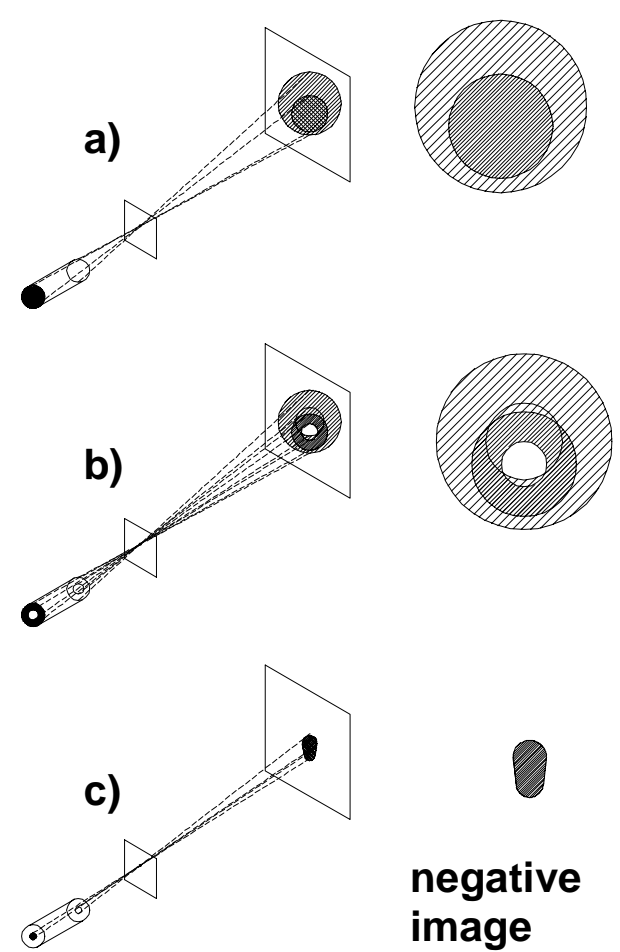

\section{negative image}

Figure 12. Qualitative images obtained with a pinhole not in the capillary axis. Images created from opposite ends of the capillary have different magnifications, and are centered on different position. a) In the case of a radial homogeneous plasma, the image corresponds to non concentric circles. b) In a hollow plasma column, the image has a more complex structure. c) In a compressed plasma, the image becomes a line [12].

Fig. 13 shows a time-integrated spectrum over ten discharges along an 8-mm length capillary (a single shot did not have enough intensity under these conditions to register a spectrum). Several lines are attributed to ArVIII , ArIX and ArX ions (second and higher orders can be identified: ArIX-2, ArIX-3, ArIX-5). The ion temperature can be roughly estimated at about $40-60$ $\mathrm{eV}$.

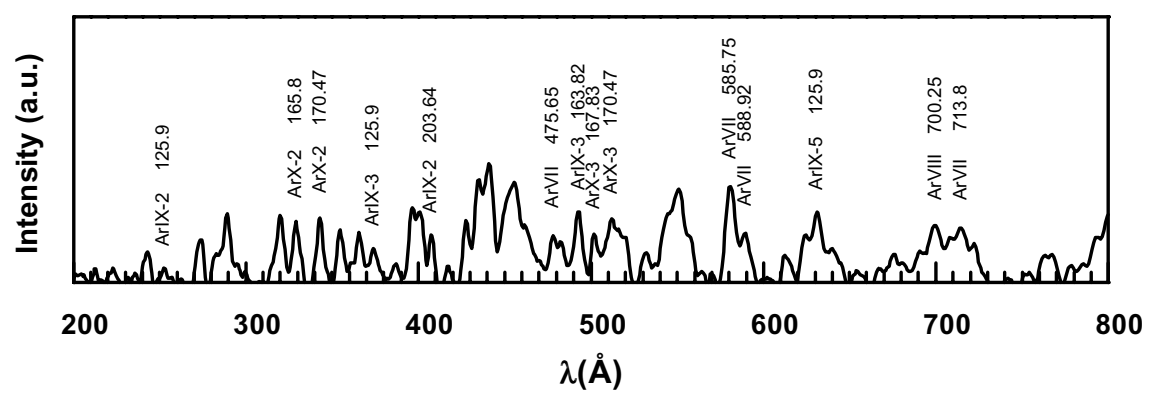

Figure 13. Time-integrated spectrum: 10 shots were registered in the same frame of the MCP for a discharge with a capillary $8 \mathrm{~mm}$ in length and $1 \mathrm{~mm}$ in diameter. 
The capillary discharge can also be complemented with numerical simulations. A one-dimensional model derived from the magnetohydrodynamics (MHD) equations was applied to study the plasma dynamics and temperature evolution during the discharges [35]. The calculations predict a final radius of 100-200 microns and electron densities of $2 \times 10^{24} \mathrm{~m}^{-3}$ occurring three nanoseconds after the peak current. The corresponding electron and ion temperatures are 80 and $40 \mathrm{eV}$. Under these conditions, theoretically, soft X-ray lasing can occur at $46.9 \mathrm{~nm}$, due to population inversion by collisional electron excitation of Ne-like ArIX ions.

\section{Quasi-static Z-pinch driven by a small generator. Early Stage, Plasma dynamics and Stability}

Z-pinch discharges are very simple devices that can produce hot dense plasmas. The limitation is the discharge disruption by instabilities. The development of pulse power generators, capable of delivering currents to a plasma load of $I>10^{5} \mathrm{~A}$ with $d I / d t>10^{12} \mathrm{~A} / \mathrm{s}$ led to a widely held belief that by heating and compressing the plasma at a high enough rate, dense hot plasma conditions could be achieved in times shorter than the instability growth time [36]. A number of experiments have been carried out in Z-pinches using pulse power generators. Results obtained show that in fiber and compressional Z-pinches $m=0$ magnetohydrodynamic (MHD) instabilities are present. On conventional gas embedded Z-pinches, after an initial expansion, $m=1$ instabilities leading to a helix develop, eventually disrupting the plasma column. The development of these instabilities happen during tens of nanoseconds. The observed growth time for the instabilities appearing in Z-pinch discharges, in both gas embedded Z-pinch [37] and fiber [38] or compressional is longer than those predicted by MHD theory. In a gas embedded Z-pinch, where an electric discharge between the electrodes is established in a dense gas under atmospheric range pressure, it is possible to obtain a large number of experiments per day in comparison with single fiber or fibers array experiments. In addition, changing the filling pressure and electrode configuration, different initial conditions and different stability regimes can be studied.

In this section we describe a series of experiments in gas embedded Z pinches carried out at PUC [14, 15, 39, 40], driven by a small pulse power generator, a Marx bank $(400 \mathrm{kV})$ coupled to a water transmission line $(1.5$ $\Omega, 300 \mathrm{kV}, 120 \mathrm{~ns}$ double transit time). The current rate was approximately $2 \cdot 10^{12} \mathrm{~A} / \mathrm{s}$. The discharges were performed in $\mathrm{H}_{2}, \mathrm{D}_{2}$ and $\mathrm{He}$ at 1/6, 1/3 and 1 bar.

The electron density profile $n_{e}$, the line electron density $N$ (number of electron per unit of length), and the pinch radius $a$, were measured with good temporal and spatial resolution. A frequency doubled Nd-YAG laser pulse (6ns) was used for optical diagnostics to obtain multiframe holographic interferometry ( 8 frames separated by $10 \mathrm{~ns}$ between them) [13], and to obtain simultaneous single shot image-plane holographic interferometry and shadowgraphy. A visible streak camera provided radial and axial plasma motion [15]. The total current and the external voltage were also measured with a Rogowskii coil and a capacitive divider.

Fig. 14 shows interferograms and current traces obtained in $\mathrm{H}_{2}$ at $1 / 3$ bar. The gas was pre-ionized using three different methods. The first method was based on laser induced ionization (Fig. 14a, laser initiated pinch). A pulse laser beam (Nd-YAG, 6 ns, $300 \mathrm{~mJ}$ ) was focused axially through the hollow anode onto the cathode a few nanoseconds before delivering the generator pulse to the electrodes. The size of the laser spark was less than 1/10 of the interelectrode distance. The experiments showed that the plasma column expands in a radial direction during the first 25 ns $\left(v_{r}=1.7 \times 10^{4} \mathrm{~m} / \mathrm{s}\right)$, and then develops $\mathrm{m}=1$ instabilities. The electron density at the axis $n_{e} \sim 10^{24} \mathrm{~m}^{-3}$ was less than the filling gas density $\left(\sim 1.8 \cdot 10^{25} \mathrm{~m}^{-3}\right)[39$, 40]. During the column expansion, the line density increased linearly $\left(4 \cdot 10^{25} \mathrm{~m}^{-1} \mathrm{~s}^{-1}\right)$. Bennett temperature $\mathrm{T}_{B}$, can be estimated from $\mathrm{T}_{B}=\left[\mu_{0} / 16 \pi \mathrm{k}(\mathrm{Z}+1)\right] \mathrm{I}^{2} / \mathrm{N}$, with $\mu_{0}$ the permeability of free space, $\mathrm{k}$ the Boltzman constant and $\mathrm{Z}$ the number of charges or ionization stages. Thus $\mathrm{T}_{B}[\mathrm{eV}]=1.56 \times 10^{11} \mathrm{I}^{2} / \mathrm{N}$, with $\mathrm{I}$ and $\mathrm{N}$ in SI units. At 25ns the Bennett temperature is estimated at $\mathrm{T}_{B} \sim 90 \mathrm{eV}$. While the plasma column expanded, the line density, $\mathrm{N}$, increased roughly in a linear way, with a rate on the order of $4 \times 10^{25} \mathrm{~m}^{-1} \mathrm{~s}^{-1}( \pm 30 \%)$. 

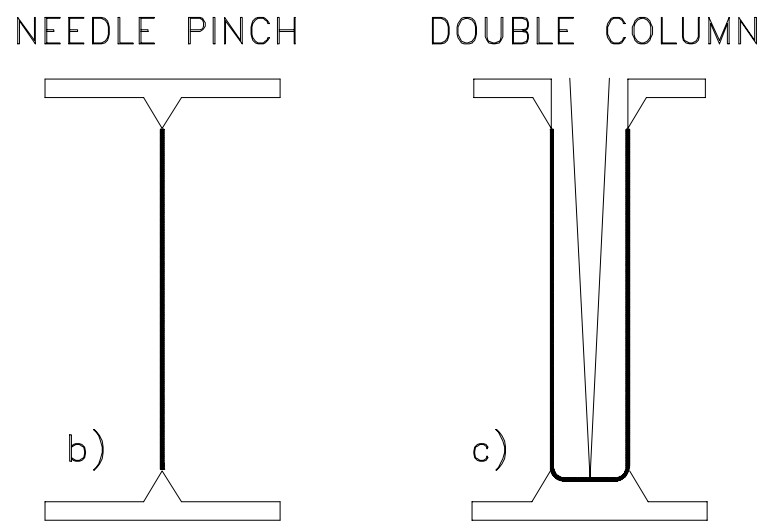

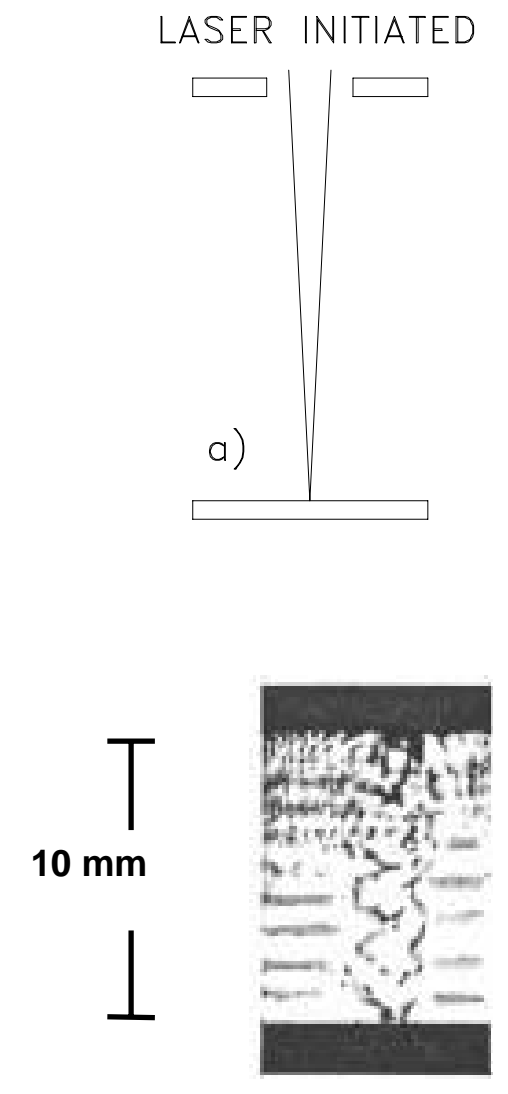

$45 \mathrm{~ns}$

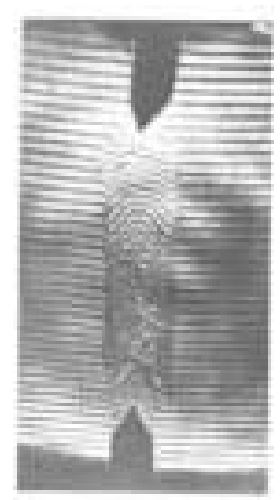

$56 \mathrm{~ns}$
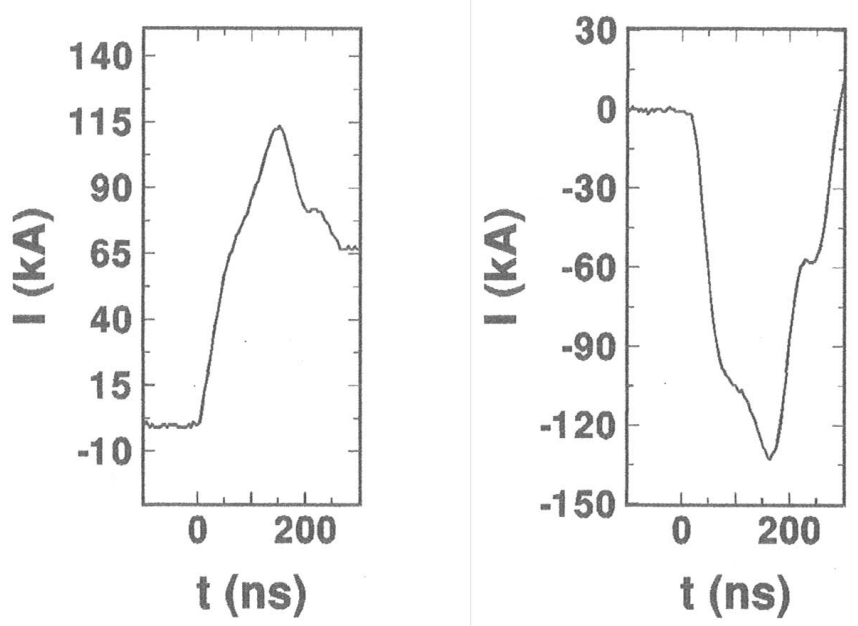

Figure 14. $\mathrm{H}_{2}$ at $1 / 3$ bar gas embedded Z-pinch experiments. a) pre-ionization by the laser pulse (laser initiated pinch), b) pre-ionization by microdischarge (needle pinch), c) pre-ionization by the hollow microdischarge and laser pulse (double column pinch). Current traces and interferograms are shown. 
For the second method, a small $20 \mu \mathrm{A}$ discharge was induced between two tungsten needle electrodes (Fig. $14 \mathrm{~b}$, needle pinch). Here the plasma column expanded $\left(v_{r}=2.6 \times 10^{4} \mathrm{~m} / \mathrm{s}\right)$ during the current rise stage. No instabilities were observed during the expansion $[39,40]$. A plume occupying $1 / 3$ of the electrodes interspace was observed at $5 \mathrm{~ns}$. An $n_{e}$ value of $5 \times 10^{25} \mathrm{~m}^{-3}$ was observed at $50 \mathrm{~ns}$ at the axis of the pinch, with a value of $\mathrm{N}$ of $\sim 1.5 \times 10^{19} \mathrm{~m}^{-1}$, and Bennett Temperature was estimated at $\mathrm{T}_{B} \sim 40 \mathrm{eV}$. Line density, $\mathrm{N}$, increased with a rate of $2.8 \times 10^{26} \mathrm{~m}^{-1} \mathrm{~s}^{-1}( \pm 30 \%)$. In this case, electron densities were greater than expected according to the density of filling gas measured.

For the third scheme, a cylindrical hollow discharge of $150 \mu \mathrm{A}$ was established between the conical electrodes; as well, a pulse laser beam was used for preionization of the central zone $[14,15]$ (Fig. 14c, double column pinch). When the pulse voltage was applied, an initial $10 \mathrm{~ns}$ fast radial expansion phase was followed by about a $50 \mathrm{~ns}$ expansion of the central channel with a velocity of $\sim 2.6 \cdot 10^{4} \mathrm{~m} / \mathrm{s}$. The expansion of the internal boundary of the annular plasma was of the same order, whereas its external boundary had a slower expanding velocity $\sim 6 \cdot 10^{3} \mathrm{~m} / \mathrm{s}$. At the end of these expansion phases, two initial plasmas coalesced into the single plasma column with an apparent internal structure that was maintained during the next 110 ns. The line density, $\mathrm{N}$, increased with a rate of the order of $1 \cdot 10^{26} \mathrm{~m}^{-1} \mathrm{~s}^{-1}$ $( \pm 30 \%)$ in the earliest $80 \mathrm{~ns}$, thus the rate decreased. The $n_{e}$ profiles after the $70 n s$, and until the current peak time, showed a central peak, with a maximum $n_{e}>4 \times 10^{25} \mathrm{~m}^{-3}$ and a value of $\mathrm{N}$ of $\sim 5 \times 10^{19} \mathrm{~m}^{-1}$, and Bennett Temperature was estimated at $\mathrm{T}_{B} \sim 40 \mathrm{eV}$. The value of $n_{e}$ was greater than what would be expected from the filling gas density. The size of the laser spark was less than $1 / 10$ of the interelectrode distance at $20 \mathrm{~ns}$, and no plasma plume was observed from the hollow electrodes. Double column pinch configuration discharges were characterized by low expansion rates and high electron densities, being in general more stable $[14,15]$.

Bennett equilibrium (quasistatic or stationary pinch) has been assumed because the Alfven velocity, $v_{A}$, was greater than the radial velocity in these three configurations.

The curious feature observed in the experiments described above was that the pinch radii always expanded ("no pinch effect"). This effect was rather surprising, and contradicts previous theoretical model calculations (see for example a zero dimensional model in refs. [41, 42]). Actually, the models mentioned were derived assuming constant electron line density, while in these experiments the line density was always increasing. It is possible to develop an extended zero dimensional model including the contributions of the plasma density in the energy balance, continuity equation and Bennett relation [39, 43], which shows an excellent agreement with the experimental data.

The relevant parameters regarding stability properties can be estimated from measured line density $N$, current $I$, and radius $a$ [44]. In fact, assuming Bennett equilibrium, Haines and Coppins [44] found for the Larmor radius over pinch radius $a_{i} / a$, for the transient Alfvén time $\iota_{A}=a / v_{A}$, where $v_{A}$ is the mean Alfvén speed, and for Lundquist number, $S$ :

$$
\begin{gathered}
\frac{a_{i}}{a}=5.7 \cdot 10^{8} \frac{1}{N^{1 / 2}}, \\
\iota_{A}=\frac{a}{v_{A}}=2.6 \cdot 10^{-10} a \frac{N^{1 / 2}}{I}, \\
S=5.46 \cdot 10^{23} \frac{I^{4} a}{N^{2}},
\end{gathered}
$$

for hydrogen in SI units. The number $\ln \Lambda=5.36$ has been used [39].

In order to determine if the plasma is in the magnetized regime the relevant parameter to examine is the product of ion cyclotron frequency $\Omega_{i}$ by collision time for the ions $\iota_{i}$.

$$
\Omega_{i} \tau_{i}=5.15 \cdot 10^{30} \frac{I^{4} a}{N^{5 / 2}},
$$

for hydrogen in SI units.

Using the experimental measurements it is possible to evaluate these parameters. For the three preionization schemes considered, from the value $\Omega_{i} \iota_{i}, 0.1$ -1 , it is apparent that the pinches are at the boundary of unmagnetized and magnetized regimes. The needle pinch and double column pinch remain stable during many transient Alfvén times $(\sim 20 \mathrm{~ns}$ at $\sim 80 \mathrm{~ns})$. It has been theoretically conjectured that there is a threshold for the stabilization due to resistive effects, corresponding to $\mathrm{S} \sim 100$. Experimentally, it has been observed that for Z-pinch discharges with a substantially lower value of $\mathrm{S}$, no instabilities appear. From the values of $\mathrm{S}$, obtained at early stage, it is apparent that the three discharges are resistive at early stages. In the laser initiated pinch case, when the instability $m=1$ starts, the value of $\mathrm{S}$ is on the order of 150 , after the value increases. The value observed for $a_{i} / a$ is at a later stage on the order of $0.1-0.2$ for the needle pinch and double column pinch. This is consistent with theoretical studies which indicate that the region of minimum instability for pinch discharges is in the neighborhood of $a_{i} / a=0.2[45,46]$.

All the various configurations studied are initially in the resistive regime. Based on experimental observations in the double column pinch and in the needle pinch [47], it would appear that a pinch could be maintained stable if it crosses over the $\mathrm{S} \sim 100$ boundary with a value $a_{i} / a$ of around $0.1-0.2$ and remained at this value while the current is increasing. 
To study the internal structure of the pinch, a onedimensional two-temperature MHD model was numerically analyzed [48]. Interestingly, the results showed that the structure of the initially pre-ionized region greatly affects pinch dynamics coinciding with the experiments described above. There is still a lot of topics for further research in small Z-pinches.

\section{Plasma focus in the limit of very low energy}

Plasma focus is a pinch discharge produced between two coaxial electrodes. The discharge starts over the insulator surface (Fig. 15a); the current sheath is magnetically accelerated along the coaxial electrodes (Fig. $15 \mathrm{~b}$ ), and after the current sheath runs over the ends of the electrodes a radial compression of the plasma occurs (Fig 15c). The final pinch generates beams of ions and electrons, and ultra short X-ray pulses. Using Deuterium gas, plasma focus devices produce fusion D-D reactions, generating a pulse of fast neutrons and protons.

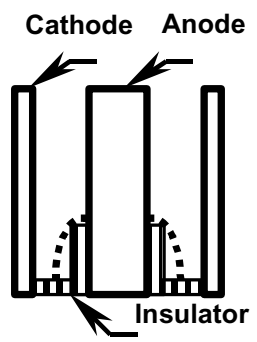

(a)

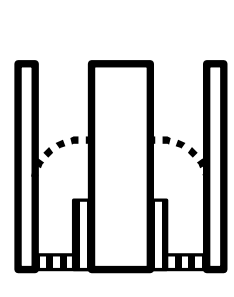

(b)

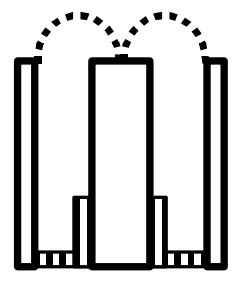

(c)

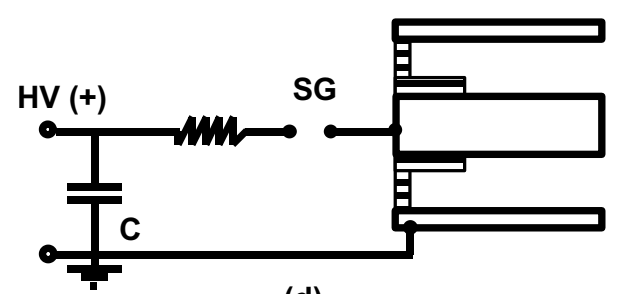

(d)

Figure 15. Schematic drawing of the principal stages in the plasma dynamics in a plasma focus device. a) The discharge starts in the base of the electrodes, a current sheath is formed at the insulator surface; b) the current sheath is accelerated along the coaxial electrodes, c) and a radial compression of the plasma occurs after the current sheaths reaches the axial end of the electrodes. d) Scheme of the circuit, a capacitor $\mathrm{C}$, is discharged over the electrode through a spark gap SG.

During the last thirty years, substantial efforts and resources have been invested in plasma focus devices [11, 49-51]. The studies range from small devices of around hundreds of Joules, to large facilities of about 1 MJ. Recently, plasma focus called the attention of the plasma research community as a mean to develop pulsed radiation applications.
Here we describe a small plasma focus operating in the limit of very low energy ( $100 \mathrm{~J}$ or less). The plasma dynamics in this limit (low capacitance) are faster (low inductance) than in large devices. Although in small plasma focus the neutron yield per pulse is quite low $\left(10^{4}-10^{5}\right)$, it is possible to operate the device in a repetitive regime from $\mathrm{Hz}$ to $\mathrm{kHz}$.

The procedure to design a small plasma focus is to start with the available electrical components. In our case we had four capacitors $(40 \mathrm{nF}, 20 \mathrm{nH})$ connected in parallel $(160 \mathrm{nF}, 5 \mathrm{nH})[52,53]$. Charging voltages of 30 to $40 \mathrm{kV}$ could be obtained from a power supply or an intermediate pulse voltage multiplier [24]. The optimum size of the electrodes was then determined by theoretical considerations, using a theoretical model of Mather plasma focus [55]. From that theoretical model the expected neutron yield for electrodes of length $\mathrm{z}=1 \mathrm{~cm}$, internal and external radii of $\mathrm{r}_{1}=0.8 \mathrm{~cm}$ and $\mathrm{r}_{2}=1 \mathrm{~cm}$, and Deuterium pressure in the range 0.1 $-1 \mathrm{mb}$ is $10^{4}-10^{5}$ for discharges operating at charging voltages from 30 to $50 \mathrm{kV}$. An external inductance of $50 \mathrm{nH}$ was assumed.

An important practical issue is the inductance of the circuit, which should be kept as low as possible. In small devices the inductance strongly depends on the length of the connections. In our case, we minimized the length of the connections between capacitor bank, spark gap and electrodes using a compact configuration. A total external inductance of $60 \mathrm{nH}$ was measured. Thus the total impedance of the generator was of the order of $0.6 \Omega$.

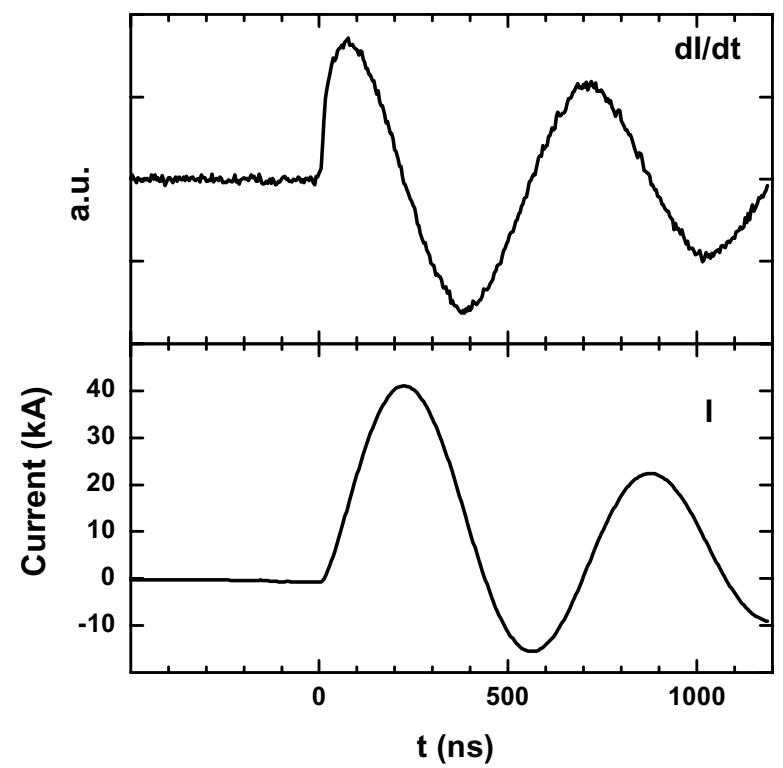

Figure 16. Electrical signals for a shot obtained in a very small Plasma Focus in hydrogen at 1 mbar at $25 \mathrm{kV}$ charging voltage (50 J energy storage in the capacitor bank). The capacitor bank of the device has a capacitance $\mathrm{C}=160 \mathrm{nF}$ and a total inductance of $\mathrm{L}=60 \mathrm{nH}$. The size of the electrodes are: length $\mathrm{z}=1 \mathrm{~cm}$, internal and external radii of $\mathrm{r}_{1}=0.8 \mathrm{~cm}$ and $\mathrm{r}_{2}=1 \mathrm{~cm}$. 


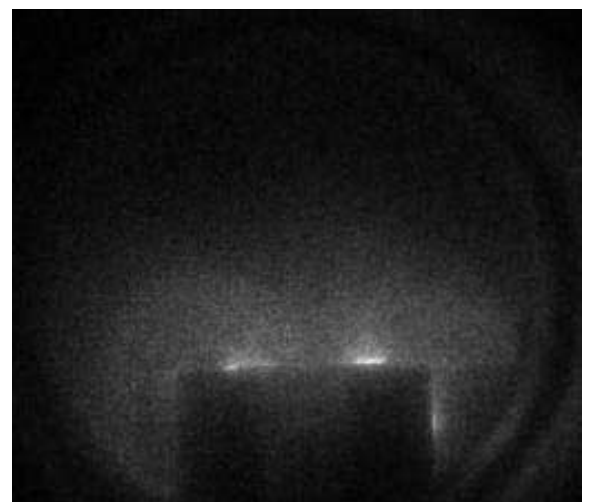

$200 \mathrm{~ns}$

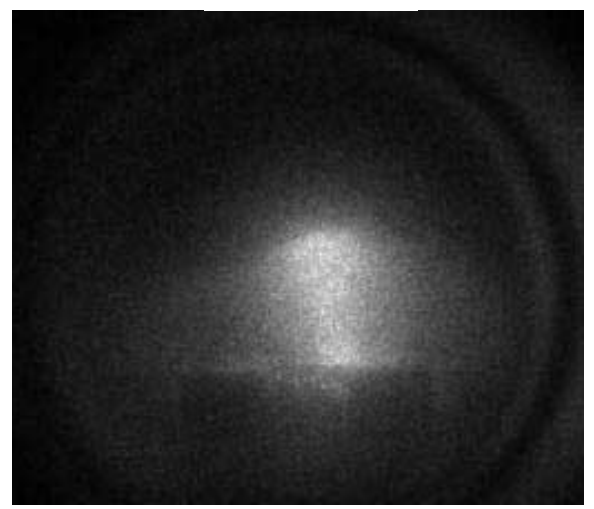

$380 \mathrm{~ns}$

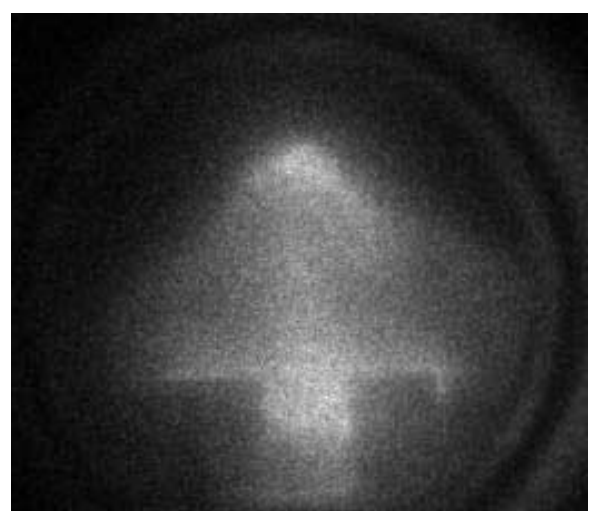

$570 \mathrm{~ns}$

\section{$20 \mathrm{~mm}$}

Figure 17. A sequence of plasma image obtained with a 5 ns gated ICCD camera for different shots in a very small Plasma Focus at the conditions of the Figure 16 is shown. After the pinch plasma column (380ns), the column disruptions can be observed (570ns). Studies in order to obtain the pinch at the moment of the peak current are carried out in the present period.

Fig. 16 shows electrical signals for a typical shot in hydrogen. Fig. 17 shows a sequence of plasma image obtained with a 5ns gated ICCD camera for different shots at the conditions of the Fig. 16. The current sheath compression is clearly observed. The typical dip in the signal of the current derivative observed in most of the plasma focus devices at the moment of the maximum compression does not appear in our experiments. Probably it is due to the impedance of the pinch is much smaller in comparison with the generator impedance. Future studies in order to obtain the pinch at the moment of the peak current will be carried out. Performance studies for neutrons emission from Deuterium plasma will be carried out in the near future.

The device constructed by us is useful both for basic research and applications. Other possible diagnostics are time integrated and time resolution neutron and X-ray detection, PIN diode and particles detectors, pulsed interferometry, among others. Potential applications are X-ray imaging, neutron interrogation of substances, and neutrography.

\section{Research in dense plasmas driven by a mega-ampere de- vice}

A recent interest for Z-pinches has aroused as sources of soft X-ray destined to irradiate targets in order to obtain thermonuclear conditions by inertial confinement [3]. The pinch is a mean to produce an ultrahigh brilliant soft X-ray source which irradiates a DeuteriumTritium target. Under this approach, the Z-pinch is not the dense plasma where thermonuclear reactions are produced as was expected during decades. These experiments are carried out in huge generators capable of delivering 1- 20MA in $100 \mathrm{~ns}\left(d I / d t>10^{14} \mathrm{~A} / \mathrm{s}\right)$ into a plasma formed from cylindrical cages of fine metallic wires. Active research on Z-pinches, are currently going on at several laboratories in the USA, England, Germany, Russia and elsewhere [4-9]. The largest facility is at the Sandia National Laboratory in the US, with huge generators capable of delivering 20MA in 100ns $\left(d I / d t>10^{14} \mathrm{~A} / \mathrm{s}\right)$ into the plasma. Similar large facilities are available at TRINITI in Russia.

Recently, the pulse power generator SPEED 2, a medium energy and large current device (187kJ, 4MA, $300 \mathrm{kV}, 400 \mathrm{~ns}, \mathrm{dI} / \mathrm{dt} \sim 10^{13} \mathrm{~A} / \mathrm{s}$ ) [11], has been transferred from the Düsseldorf University to the Plasma Physics Group of the Comisión Chilena de Energía Nuclear The SPEED 2 arrived at the CCHEN in May 2001 and will be in operation by January 2002, being the most powerful and energetic device for dense transient plasma in the Southern Hemisphere. Experiments in different Z-pinch configurations using the SPEED 2 generator will be carried out at CCHEN in the future. A medium size energy device provides a good opportunity to obtain measurements that are not possible to reach in large devices, where the huge energy release in the load region requires heavy shielding, and forces one 
to locate the diagnostics equipment at large distances from the pinch area.

A device designed 15 years ago, as SPEED 2, can be used for the research of scientific topics relevant today and for the research and development of new ideas. Considering the world frame, three open subjects in the physics of dense plasma pinches will be studied at CCHEN in the future: a) High brightness and soft Xray radiation from transient electrical discharges. The plasma will be obtained from a plasma focus and wires array (using the SPPED 2 generator), and capillary discharge configuration (using small devices). Special interest to identify conditions for population inversion in transient plasmas in order to obtain high brightness VUV to soft X-ray radiation and X-ray lasers; b) Neutron flux characteristics from plasma focus discharges operating in Deuterium (with temporal and spatial resolution) correlated to discharge parameters, plasma dynamics and instability. Particular investigation of the effect of insulator surface preparation and conditioning on pinch behavior and neutron yield; and c) Magnetic confinement in a quasistatic z-pinch at mega ampere current peak. Plasma dynamics and stability in an original quasi-static z-pinch configuration proposed by L. Soto (a gas embedded compressional Z-pinch, see section IV and references $[14,15]$ ) will be studied at high current using the SPEED 2 generator.

The laboratory plasmas proposed to be studied will be driven by SPEED 2 generator, working at hundred of $\mathrm{kA}$ to MA current peak regimes, delivering at current rates $10^{12}-10^{13} \mathrm{~A} / \mathrm{s}$. The SPEED 2 device will allow to perform the future program by extending previous research in small devices and completing them with a wider span of experiments.

Most of the previous works developed in SPEED 2 at Düsseldorf were done in a plasma focus configuration. The Plasma Physics Group at University of Düsseldorf provides technical assistance and direct support for setting-up and putting in operation the SPEED 2 locally in Chile. The Chilean operation will begin implementing and developing diagnostics in a conventional plasma focus configuration. Then, after getting the experimental expertise with SPEED 2, new experiments in the quasi-static Z-pinch and wires array will be developed to extend the device capabilities.

Some specific topics that will be studied using the SPEED 2 at CCHEN are the following:

Instabilities and neutron emission. The origin of the neutron emission from a plasma focus operating in Deuterium is attributed mainly to two mechanism: a) thermonuclear reactions and b) beam-target reactions [see for example 49, 56-57]. The second mechanism is associated to ions acceleration processes in a $\mathrm{m}=0$ instability and the subsequent gap developed in the plasma column [7], similar to early Z-pinch experiments. Many authors indicate that this would explain the anisotropy observed in spatial distributions of the emitted neutrons. Similar instabilities occur in cryogenic Deuterium fiber discharges, where diagnosed experiments, with current rising to $1 \mathrm{MA}$ in 200ns, show that disruptions can occur at late stages, leading to relativistic electron beams, which are associated to hard $\mathrm{X}$-rays burst and neutrons yield. However, this is a controversial area for theory.

SPEED 2 experiments for neutron flux characterization (with temporal and spatial resolution), operating in a plasma focus configuration filled with Deuterium gas, will be carried out. The aim is to correlate discharge parameters, plasma dynamics and instability. It is proposed to attempt to establish an experimental correlation between the spatial anisotropy of the neutron emission and total yield with hard X-ray emission and hot spot formation. Particularly, the interest will also involve a research on the effect of the preparation and conditioning of the insulator surface on pinch behavior and neutron yield.

We will intend to find net results related with neutron pulse emission processes, and plasma properties loci where they are being generated (linked to the hot spot), to establish validity range with theoretical models. The results will be outstanding from the transient dense plasma physics perspective, and they will contribute to establish design criterion of plasma focus optimized devices in order to generate pulsed neutron beams with potential applications in detection and analyze systems.

In addition, to separate the two principal neutron production mechanisms in a Deuterium pinch plasma (thermonuclear and the so-called beam-target), it is proposed to establish a comparison between measurements of neutron spatial distributions in a plasma focus (in which a $\mathrm{m}=0$ instability occurs), and measurements of neutron spatial distribution from a stable z-pinch to a $\mathrm{m}=0$ mode, under similar density and temperature parameters. A quasi-static Z-pinch as the gas embedded compressional z-pinch $[14,15]$, as described in the previous section IV, operating in Deuterium with current rising to $\sim 1 \mathrm{MA}$ in $\sim 200 \mathrm{~ns}$ is a candidate to this experimental proposal driven by SPEED2.

Quasi-static Z-pinch. A quasi-static and stable Zpinch in order to obtain a dense and hot plasma for thermonuclear fusion applications is a foundational goal for the Z-pinch research.

The dynamics and stability of the double column pinch $[14,15]$, described in the previous section III, will be studied at high current in the SPEED 2. The initial impedance of the double column pinch is compatible with the SPEED 2 impedance in order to obtain a maximum current about $1 \mathrm{MA}$ or higher. That is not the case for a single fiber, where a maximum current of $700 \mathrm{kA}$ was obtained in previous experiments in Düsseldorf. So, once again, a device designed 15 years ago, can be used for the research and development of new ideas. 
It is interesting to note that the geometry of this configuration, two coaxial column, is the geometry used in Sandia experiments for wires arrays. This geometry provides the stability observed in experiments of Sandia Laboratory. A gas embedded z-pinch allows to study different stability regimes by changing the filling pressure.

The neutron spatial distribution from a gas embedded compressional Z-pinch, operating in Deuterium, will also be measured and compared to the neutron spatial distribution from a plasma focus with a comparable density and temperature.

Z-pinch as intense $X$-ray sources. X-ray radiation is emitted from high energy density plasma generated by pinching phenomenon. The $\mathrm{Z}$ accelerator (11.4 MJ, 20MA, 100ns) at Sandia Natinal Laboratory, USA, is the most powerful X-ray source in the world. A current of $20 \mathrm{MA}$ rising in 100ns over a tungsten wire arrays produce a pinching plasma that emits a X-ray pulse (10ns FWMH ) with a peak power of 200 TW [4-6] . The final goal of that mega-project is to produce inertial confinement fusion irradiating D-T targets using the VUV radiation emmited from the Z-pinch plasma (hohlraum device) [6]. Even tough at Sandia huge powerful X-ray pulse have been obtained, the related physical mechanisms are yet an open field.

For high-temperature plasmas the bulk of the emission spectrum is distributed in VUV and soft X-ray regions and its application in various fields is being expected. Also, hard X-ray emission is observed during the discharge. The physical mechanism related with these emissions are an open field yet, both experimentally and theoretically. Radiative collapse [41, 42] and population inversion are interesting phenomena. Radiative collapse occurs if the power radiation losses are greater than the input power on the plasma. A hot (hundred of eV), small size (microns to submicrons sizes) and high density (solid density) plasma is predicted in theory. On the other hand, population inversion of ions is a necessary mechanism to produce laser emission [29, 59]. Experimental results on VUV lasing have been reported by Rocca et al. [27] and recently by other researchers [60]. However, the phenomenology and physical mechanism are also an open field. The key point to produce those conditions is the current rate, which should reach about $10^{13} \mathrm{~A} / \mathrm{s}$. What are the proper combinations between current derivative and peak current in order to obtain population inversion in a soft X-ray region? For a large dI/dt, which is the minimum peak current to produce population inversion in the VUV to soft X-ray emission? Is it experimentally achievable?

\section{Conclusions}

A review of the dense transient plasmas researches, developed by the Chilean Nuclear Energy Commission, has been presented. Previous published results have been shown and properly referenced. Recent new results have been sketched along this article.

Three kinds of pinch discharges driven by small devices were presented. The devices are relatively easy to construct and do not require large budgets, being suitable for small plasma laboratories.

The capillary discharge is a clever method to obtain high power densities with low energy. The experiment raises interesting technological questions that have not yet been answered: Is there an optimum combination of current derivative and peak current to obtain population inversion in the soft $\mathrm{X}$-ray region, and if so, is it experimentally achievable?

Gas embedded Z-pinch experiments are useful to study pinch dynamics and instabilities. Peak currents of tens of $\mathrm{kA}$, with current derivatives of $10^{12}-10^{13} \mathrm{~A} / \mathrm{s}$ could be achieved in small devices. Experiments in small Z-pinches are interesting for the study of the earliest stages of plasma discharges. However, diagnostics with sub-nanoseconds resolution within tens of micrometers are still required. Future studies in a gas embedded compressional Z-pinch driven by a mega-ampere device (SPEED 2) was discussed.

Neutron generation from plasma focus devices has been a matter of research in the past. Nowadays, the technology is focused on transportable neutron generators for in situ applications (neutron illumination, neutrography, and substance detection). Moreover, intense neutron pulses have interesting applications in biology and material sciences. Plasma-focus pulses also show other unique technological features, namely simultaneous combination of X-ray and neutron emissions, which can be used to study compound mixing of high and low atomic numbers. On the other hand, it is interesting to study if the existing theoretical models are valid in the very low energy limits. Although small plasma devices are relatively easy to construct, plasma diagnostics remain difficult. High spatial and temporal resolution diagnostics are required. In order to obtain the maximum benefit from these small devices, proper instruments and diagnostics must be developed.

An important goal of the plasma physics group of the CCHEN has been to develop plasma diagnostics and instrumentation. Two of the described experiments were constructed in the Comisión Chilena de Energía Nuclear, CCHEN. In order to study the generated plasmas in these two devices, the following diagnostics were developed and applied: fast voltage, current and current derivative monitor; time and space resolved pinhole images; time resolved VUV spectroscopy; time integrated multipinhole X-ray photography; and plasma images using a gated intensified CCD camera. A sys- 
tem for optical refractive diagnostics as shadowgraphy, schlieren and interferometry using a ICCD camera gated from 3 to $20 \mathrm{~ns}$ in combination with a continuous He-Ne laser, is ready to be applied.

To the diagnosis capability achieved at the CCHEN, we will add in the near future: 1ns time resolution holographic interferometry with a $\mathrm{N}_{2}$ laser; 2 dimensional optical multichannel analyzer; time integrated and temporal resolution neutrons detection; time resolved broad band emission using filtered PIN diode arrays; and time resolved X-ray spectroscopy.

Future experiments driven by a medium energy and large current device (SPEED 2, 4MA, 187kJ, 400ns, $\mathrm{dI} / \mathrm{dt} \sim 10^{13} \mathrm{~A} / \mathrm{s}$, that will be in operation since January 2002 at CCHEN) has been discussed. Experiments in plasma focus, quasi-static z-pinch and wires array are being planed.

The combination of the proposed diagnostics applied to plasmas driven by small and mega- ampere devices, will allow us to perform accurate measurements of plasma dynamics, radiation and particle emission. As an overall result, valuable experimental information will be available in order to be compared with theoretical models for dense plasmas.

\section{Acknowledgement}

The authors acknowledge the support from the Plasma Physics Group of the Pontificia Universidad Católica de Chile during the end of 1993 up to the beginning of 1998. At the present there are active collaborations with the Network PLADEMA (Argentina) and with the Comisión Nacional de Energía Atómica; and with the Plasma Spectroscopy Group of Institute of Spectroscopy, Russian Academy of Science, Troitsk, Russia. An important collaboration has started with the Plasma Physics Group of University of Düsseldorf, Germany. Recently the Univesrity of Düsseldorf transferred de SPEED 2 generator to the Plasma Physics Group of the CCHEN.

Particularly the authors acknowledge the fruitful discussions with: H. Chuaqui of Pontificia Universidad Católica de Chile; A. Clausse of Network PLADEMA (Argentina), C. Moreno of Universidad de Buenos Aires (Argentina), H. Bruzzone of Universidad de Mar del Plata (Argentina); K.N. Koshelev, P. Antsiferov and A. Nazarenko of Institute of Spectroscopy, Russian Academy of Science, Troitsk, Russia; Prof. W. Kies and Prof. G. Decker of Universidad of Düsseldorf (Germany).

This work has been funded by the grant Cátedra Presidencial en Ciencias, awarded to L. Soto by the Chilean government, grants 1950058 and 1980187 Fondo Nacional de Investigación Científica y Tecnológica FONDECYT (Chile), Bilateral Agreement: Comisión Nacional de Energía Atómica (Argentina) Comisión Chilena de Energía Nuclear (Chile), and International Atomic Energy Agency, IAEA.

\section{References}

[1] D. D. Ryutov, M. S. Derzon, M. K. Matzen, Rev. of Modern Phys. 72, 167-223 (2000).

[2] M. A. Liberman, J. S. De Groot, A. Toor, R. B. Spielman, "Physics of High-Density Pinch, SpringerVerlag, 1998.

[3] C. G. Yonas, "Fusion and the Z-pinch", Scientific American, August, 1998, p. 40; J. Chitenden, "The Z-pinch approach to fusion", Physics World, May 2000, p. 39

[4] R. B. Spielman et al, Physics of Plasmas, 5, 2105 (1998)

[5] R B Spielman et al., Plasma Phys. Control. Fusion 42, B157(2000)

[6] M. Cuneo et al., Physics of Plasmas 8,. 2257 (2001)

[7] M. G. Haines, S. V. Lebedev, J. P. Chittenden, F. N. Beg, S. N. Bland, and A. E. Dangor, Physics of Plasmas 7, $1672(2000)$

[8] J. P. Chittenden, S. V. Lebedev, S. N. Bland, F. N. Beg, and M. G. Haines, Physics of Plasmas, 8, 2305 (2001)

[9] J. P. Chittenden, S. V. Lebedev, S. N. Bland, A. Ciardi, and M. G. Haines, Physics of Plasmas, 8, 675-678 (2001)

[10] L. Soto, A. Esaulov, J. Moreno, P. Silva, G. Sylvester, M. Zambra, A. Nazarenko, and A. Clausse, Physics of Plasma 8, 2572 (2001).

[11] G. Decker, W. Kies, M. Mälzig, C. Van Valker and G. Ziethen, Nucl. Instrum. and Methods, A249, 477 (1986).

[12] L Soto y H Chuaqui, "Sistemas para Diagnóstico Interferométrico de un Z-pinch Denso con Resolución Espacial y Temporal", Actas V Simposio Chileno de Física Experimental y Aplicada (Antofagasta, Chile) (1994).

[13] L. Soto, H. Chuaqui and M. Skowronek, Applied Optics 34, 7831 (1995).

[14] L. Soto, H. Chuaqui, M. Favre, and E. Wyndham, Phys. Rev. Lett. 72, 2891 (1994).

[15] L. Soto, H. Chuaqui, M. Favre, R. Saavedra, E. Wyndham, M. Skowronek, P. Romeas, R. Aliaga-Rossel and I. Mitchell, IEEE. Trans. Plasma Science 26, 1179 (1998).

[16] H. Chuaqui, M. Favre, E. Wyndham, R. Aliaga-Rossel, Ian Mitchell and L.Soto, , Physics of Plasmas 2, 3910 (1995).

[17] H. Chuaqui, M. Favre, R. Saavedra, E. Wyndham, P. Choi, C. Dumitrescu-Zoita, L. Soto, R. Aliaga Rossel and I. Mitchell, Physics of Plasma 4, 3696 (1997).

[18] H. Chuaqui, M. Favre, R. Saavedra, E. Wyndham, L. Soto, P. Choi, and C. Dumitrescu-Zoita, IEEE. Trans. Plasma Science 26, 1162 (1998).

[19] L. Soto and H. Chuaqui, Meas. Sci. Technol. 11, 39 (2000).

[20] L. Soto, H. Chuaqui and R. Saavedra, Meas. Sci. and Technology 8, 875 (1997). 
[21] H. Chuaqui, M. Favre, R. Saavedra, E. Wyndham, P. Choi, C. Dumitrescu-Zoita, and L. Soto, "Space and Time Resolved Observations of Hot Spot Dynamics in a Vacuum Spark Discharge", $11^{\text {th }}$ International Conference on High-Power Particle Beams, BEAMS'96 Proc. (Praga, República Checa, 1996).

[22] L. Soto, A. Esaulov, P. Silva, G. Sylvester, J. Moreno, M. Zambra and A. Nazarenko, "Plasma Dynamics and VUV Spectroscopy Diagnostics in a Fast Capillary Discharge", IX Latin American Workshop on Plasma Physics, La Serena, Chile, 2000,. AIP Conf. Proc. 563, p. 264, American Institute of Physics (2001).

[23] L. Soto, P. Silva, G. Sylvester, J. Moreno, A. Esaulov and A. Nazarenko, "Fast Capillary Discharge: Plasma Dynamics and VUV Spetroscopy Diagnostics", Rev. Mex. Fis. in press.

[24] L. Soto and L. Altamirano, Rev. Sci. Instrum. 70, 1891 (1999).

[25] I. A. D. Lewis, Elect. Eng. 27, 332 (1955).

[26] P.S. Antsiferov, L.A. Dorokhin, E.Yu. Khautiev, Yu.V.Sidelnikov, D.A. Glushkov, I.V. Lugovenko, and K.N.Koshelev, J. Phys. D: Appl. Phys. 31, 2013 (1998).

[27] J.J. Rocca, V. Shlyaptsev, F.G. Tomasel et al, Phys. Rev. Lett. 73,2192 (1994).

[28] C.H. Moreno, M.C. Marconi, V.N. Shlyaptsev, B.R. Benware, C.D. Macchietto, J.L.A. Chilla, and J.J. Rocca, Phys. Rev. A 58, 1509 (1998).

[29] J. J. Rocca, Rev. Sci. Instrum., 70, 3799-3827 (1999).

[30] P. Choi, J. G. Lunney, A. Engel, C. Dumitrescu-Zoita, T. N. Hansen, I. Krisch, J. Larour, J. Rous, "A $10^{13} \mathrm{~A} / \mathrm{s}$ High Energy Density Microdischarge Radiation Source", Proceedings IEEE Pulse Power Conference, p. 287 (published by Institute of Electrical and Electronics Engineers, Piscataway, NJ, USA, 1999).

[31] P. Choi and M. Favre, Rev. Sci. Instrum. 69, 3118, (1998).

[32] P. Choi, H. Chuaqui, M. Favre, and E. Wyndham, IEEE Trans. Plasma Sci. 15, 428 (1987).

[33] H. Chuaqui, M. Favre, L. Soto, and E. Wyndham, IEEE Trans. Plasma Sci. 21, 778 (1993).

[34] K. N.Koshelev, private communication, Troitsk, Russia, July 2000.

[35] A. Esaulov, P. Sasorov, L. Soto, M. Zambra and J. Sakai, Plasma Physics and Controlled Fussion, 43, 571 (2001).

[36] 12.- R. F. Post, Rev. Mod. Phys. 28, 338 (1956).

[37] P. Choi, M. Coppins, A. E. Dangor, and M. B. Favre, Nuclear Fusion 28, 1771 (1988).

[38] J. Sethian, A. E. Robson, K. A. Gerber and A. W. De Silva, Phys. Rev. Lett. 59, 892 (1987).

[39] L. Soto, Ph. D. thesis, Pontificia Universidad Católica de Chile, Santiago de Chile, 1993.

[40] H. Chuaqui, L. Soto, M. Favre, and E. Wyndham, in Proceedings of the $3^{\text {rd }}$ International Conference on Dense Z-Pinches (London, UK, 1993), AIP Conf. Proc. ed. M. Haines p. 27. Published by American Institute of Physics, Melville, NY.
[41] A. E. Robson, Nuclear Fusion, 28, 2171 (1988).

[42] M. G. Haines, Plasma Physics and Controlled Fusion, 31, 759, (1989).

[43] L. Soto y A. Clausse, "Evolution of a Gas Embedded Z-pinch at Atmospheric Pressure Range: A Zero Dimensional Model", submitted to IEEE Transactions on Plasma Science.

[44] M. G. Haines, and M. Coppins, Phys. Rev. Lett. 66, $1462(1991)$

[45] T. D. Arber, M. Coppins, and J. Scheffel, Phys. Rev. Lett., 72, 2399 (1994).

[46] T. D. Arber, P. G. Russell, M. Coppins, and J. Scheeffel, Phys. Rev. Lett., 74, 2698 (1995).

[47] L. Soto, H. Chuaqui, M. Favre, and E. Wyndham, in Proc. Int. Conf. on Plasma Phys. ICPP, 1994, Foz do Iguazú, Brazil, 1994, p. 216.

[48] A. Esaulov, P. Sasorov, L. Soto and M. Zambra, Physics of Plasmas, 8, 1395 (2001).

[49] R. Aliaga-Rossel and P. Choi, IEEE Trans. Plasma Sci., 26, 1138 (1998).

[50] M. Favre, P. Silva, P. Choi, H. Chuaqui, C. Dumitrescu-Zoita, and E. S. Wyndham, IEEE Trans. Plasma Sci., 26, 1154 (1998).

[51] S. Lee, T. V. Tou, S. P. Moo, M. A. Eissa, A. V. Golap, K. H. Kewk, S. Mulyodrone, A. J. Smith, Suryad, W. Usada, and M. Zakaullah, Amer. J. Phys. 56, 62 (1988).

[52] P. Silva, L. Soto, G. Sylvester, M. Zambra, H. Bruzzone, A. Clausse, and C. Moreno, "Design and Construction of a Very Small Plasma Focus in the Limit of Low Energy", IX Latin American Workshop on Plasma Physics, La Serena, Chile, 2000,. AIP Conf. Proc. 563, p. 235, American Institute of Physics (2001).

[53] P. Silva, L. Soto, G. Sylvester, M. Zambra, H. Bruzzone, A. Clausse, and C. Moreno, "Plasma Focus in the Limit of Low Energy", Rev. Mex. Fis. in press.

[54] P. Silva, L. Soto, J. Moreno, G. Sylvester, M. Zambra, L. Altamirano, H. Bruzzone, A. Clausse, and C. Moreno, "A Plasma Focus Driven by a Capacitor Bank of Tens of Joules" submitted to Review of Scientific Instruments.

[55] Moreno, H. Bruzzone, J. Martinez and A. Clausse, IEEE Trans. Plasma Sci. 28, 1735 (2000).

[56] M. J. Bernstein and F. Hai, Phys. Lett. A31, 317 (1970).

[57] V. V. Vikhrev, Sov. J. Plasma Phys. 12, 262 (1986).

[58] F Castillo, M Milanese, $\mathrm{R}$ Moroso and J Pouzo, J. Phys. D: Appl. Phys. 33 , 141 (2000).

[59] K. N. Koshelev and H. J. Kunze, Quantum Electronics 27, 164 (1997)

[60] Private communication with A. Fisher (Israel), Quebec, Canada, October 2000. 